\title{
Celiac disease presenting as primary glossopharyngeal neuralgia
}

\author{
Francis X O'Brien* and Katherine S Pickett \\ University Internal Medicine, Department of Internal Medicine, Wake Forest School of Medicine, One Medical Center Boulevard, Winston-Salem NC 27157, USA
}

\section{Dear editor}

We read with interest Dr. Khosro Farhad's article titled "Current diagnosis and treatment of painful small fiber neuropathy" [1]. In Table 1 of his article Dr. Farhad provides a comprehensive list of possible etiologies for small fiber neuropathy. This type of review gives general practitioners such as ourselves a fighting chance in making a proper diagnosis as to the etiology of small fiber neuropathy when it is encountered in the office setting. One of the possible etiologies listed is celiac disease. The awareness of celiac disease and non-celiac gluten sensitivity has increased dramatically in the last few years [2]. Your readers may be interested in the possible association of celiac disease and primary cranial nerve disorders.

A healthy 24-year-old woman presented with severe malaise and fatigue associated with left neck, chest, shoulder and arm pain after running a half marathon in April 2018. She was treated symptomatically with NSAIDs and physical therapy. Her symptoms improved but persisted. Nine months later in November 2018 she developed leftsided pulsatile tinnitus, a clicking sensation in her left ear, and a metallic taste in her mouth. CT angiography of the neck excluded carotid artery dissection. MRIs of the brain and cervical spine were normal. Her symptoms progressed to include numbness of the soft palate on the left side and sudden lightening-like spasms of pain in her throat associated with aspiration apprehension when drinking fluids. She was seen by a neurologist who noted failure of the left side of the palate to elevate with induced gag. A diagnosis of primary glossopharyngeal neuralgia was made and the patient was treated with gabapentin. The cause of her left neck, shoulder, chest and arm pain was uncertain.

The patient had partial mitigation of her symptoms with gabapentin in terms of the sharp pains in her throat and the choking sensation when drinking fluids, but her soft palate remained numb. She developed new symptoms of fleeting shocks of pain over her chest and extremities. Her left arm felt weak intermittently. In May 2019 she developed an acrid taste in her mouth that made it difficult to eat. In June 2019 she started having non-exertional symptoms of shortness of breath when lying down at night to go to sleep.

She did not appear to have a systemic illness like vasculitis or rheumatoid arthritis or malignancy. It was considered that she might have multiple lower cranial nerves involved because of her regional shoulder pain and shortness of breath. In addition, mono-neuritis multiplex was considered because of the noncontiguous neuropathic lightning-like shocks of pain in her extremities.

For the sake of diagnostic completeness she had an anti-tissue transglutaminase IgA assay to screen for celiac disease. There was little expectation that it would be helpful as the patient had no GI symptoms whatsoever. The anti-tissue transglutaminase IgA level was mildly elevated. In September 2019 the patient underwent upper endoscopy and duodenal biopsy which was suggestive of celiac disease. Her HLA DQ 8 was positive.

The patient was instructed to follow a strict gluten-free diet. Her anti-tissue transglutaminase level returned to the normal range and by December 2019 all her symptoms had resolved and she felt completely well.

There are multiple identifiable causes of glossopharyngeal neuralgia such as granulomatous disease, autoimmune or inflammatory processes, infectious or malignancy-related causes, trauma and compression [3]. The cause is idiopathic in a majority of cases. If a causation cannot be identified then a diagnosis of primary glossopharyngeal neuralgia is made.

Treatment for primary glossopharyngeal neuralgia is directed at ameliorating the symptoms with pharmacotherapy and searching for an arterial loop compressing the nerve along its anatomical course from the medulla to the jugular foramen. Severe symptoms that are unresponsive to medical therapy may warrant consideration of microvascular decompression, radiofrequency ablation of the nerve, or rhizotomy [4].

As primary care practitioners, it is gratifying to think that a treatable and previously unreported cause of a glossopharyngeal neuralgia may have been identified. We ask that your readers decide for themselves whether it would be prudent to screen patients with primary cranial nerve disorders for celiac disease.

\section{References}

1. Farhad K (2019) Current diagnosis and treatment of painful small fiber neuropathy Curr Neurol Neurosci Rep 19: 103

2. Leonard MM, Sapone A, Catassi C, Fasano A (2017) Celiac disease and nonceliac gluten sensitivity: A review. JAMA 318: 647-656.

3. Finsterer J, Grisold W (2015) Disorders of the lower cranial nerves. J Neurosci Rural Pract 6: 377-391.

4. Blumenfeld A, Nikolskaya G (2013) Glossopharyngeal neuralgia, Curr Pain Headache Rep 17: 343.

${ }^{\star}$ Correspondence to: Francis X. O’Brien, University Internal Medicine, Department of Internal Medicine, Wake Forest School of Medicine, One Medical Center Boulevard, Winston-Salem NC 27157, USA, E-mail: fobrien@wakehealth.edu

Received: June 10, 2020; Accepted: June 17, 2020; Published: June 19, 2020

Copyright: (C2020 O'Brien FX. This is an open-access article distributed under the terms of the Creative Commons Attribution License, which permits unrestricted use, distribution, and reproduction in any medium, provided the original author and source are credited. 